Reviews

Juntendo Medical Journal

2018. 64 (Suppl 1), 122-127

\title{
Evaluation of Cartilage and Bone Metabolism in Collegiate Athletes Belonging to Various Sports Clubs by Analyzing Type II Collagen Degradation and Synthesis, and Type I Collagen Degradation
}

\author{
ISAO NAGAOKA*1), AKIFUMI TSURUTA*2), MASAFUMI YOSHIMURA*3) \\ *1) Department of Host Defense and Biochemical Research, Juntendo University Graduate School of Medicine, Tokyo, \\ Japan, *2) Koyo Chemical Co. Ltd., Tokyo, Japan, *3) Juntendo University Graduate School of Health and Sports Science, \\ Chiba, Japan
}

\begin{abstract}
In this study, to evaluate cartilage and bone metabolism in athletes, the levels of type II collagen degradation marker CTX-II and synthesis marker CPII, and type I collagen degradation marker NTx were measured using urine samples of collegiate athletes belonging to various sports clubs (soccer, tennis, triathlon, squash, swimming, volleyball, kendo, judo, gymnastics, basketball, handball, baseball, long-distance, throwing-event, jumping, sprint and futsal) and compared with those of non-athlete controls ( $5 \sim 11$ subjects in each group).

NTx levels were significantly increased in soccer, volleyball, basketball and handball players compared with non-athletes. Similarly, CTX-II levels were significantly increased in soccer, volleyball and handball players compared with non-athletes. In contrast, CPII levels were significantly increased in squash players and long-distance runners compared with non-athletes. Moreover, CTX-II/CPII ratios were increased in soccer, volleyball, basketball and handball players compared with non-athlete control, suggesting that type II collagen degradation is relatively increased compared with type II collagen synthesis in these players. Together these observations indicate that cartilage and bone metabolism (type II and type I collagen degradation) is enhanced in players of ball games associated with jumping action, such as soccer, volleyball, basketball and handball.
\end{abstract}

Key words: cartilage and bone metabolism, athletes, biomarker, type II collagen, type I collagen

\section{Introduction}

The frequency and severity of joint loading are critical factors for the development of joint destruction, characterized by the damage of articular cartilage. In fact, excessive loading on the joint with motion and exposure causes the damage of articular cartilage $^{1)-4}$. Thus, sports with repetitive impact and torsional loading on the joints increase the risk of articular cartilage degeneration, and results in the clinical symptoms of osteoarthritis ${ }^{4)}$.

The disease process of osteoarthritis is related to the degradation and functional loss of articular cartilage. Importantly, the early changes in the metabolic and biochemical properties of cartilage matrix can be detected before the appearance of morphological changes of cartilage ${ }^{2)}$. Thus, various biomarkers have been developed as indicators of cartilage and bone metabolism in subjects with joint and bone disorders ${ }^{5)}$. In this context, it is interesting to note that sports-related mechanical loading on the joints affects the turnover rate of cartilage as well as bone in humans, and these changes can be detected by the assays with biomarkers ${ }^{1)-4}$.

Corresponding author: Isao Nagaoka

Department of Host Defense and Biochemical Research, Juntendo University Graduate School of Medicine

2-1-1 Hongo, Bunkyo-ku, Tokyo 113-8421, Japan

TEL: +81-3-5802-1032 FAX: +81-3-3813-3157 E-mail: nagaokai@juntendo.ac.jp

〔Received Oct. 23, 2017〕〔Accepted Feb. 13, 2018〕

Copyright (C) 2018 The Juntendo Medical Society. This is an open access article distributed under the terms of Creative Commons Attribution License (CC BY), which permits unrestricted use, distribution, and reproduction in any medium, provided the original source is properly credited. doi: 10.14789/jmj.2018.64.JMJ18-P51 


\section{Biomarkers for cartilage and bone metabolism}

Type II collagen is one of the major components of cartilage ${ }^{6}$, and the fragments of type II collagen are utilized as biomarkers for cartilage metabolism. A C-terminal telopeptide (CTX-II) is cleaved during degradation of type II collagen ${ }^{7)}$, whereas a neo-epitope $(\mathrm{C} 2 \mathrm{C})$ is cleaved at the $\mathrm{C}$ terminus of the $3 / 4$ piece of degraded type II collagen ${ }^{8)}$. Thus, both CTX-II and C2C are used as markers for type II collagen degradation. In contrast, a C-terminal type II procollagen peptide (CPII) is present in newly formed type II procollagen and cleaved during processing of synthesized type II procollagen; thus, CPII can be used as a marker for type II collagen synthesis ${ }^{9}$. In addition, deoxypyridinoline (Dpyr), a crosslink product of type I collagen and a cross-linked $\mathrm{N}$-terminal telopeptide of type I collagen (NTx) are used as markers for type I collagen degradation in bone (bone resorption) ${ }^{5}$.

\section{Effect of endurance exercise on cartilage and bone metabolism}

It has been already reported that sports and exercise affect cartilage and bone metabolism.
O'Kane et al. compared the urine levels of type II collagen degradation marker CTX-II and type I collagen degradation marker NTx among nonathlete controls, cross-country runners, swimmers and crew members ${ }^{3)}$. The results indicated that the levels of CTX-II and NTx are increased in the cross-country runners and crew members compared with non-athletes and swimmers, suggesting that cartilage and bone metabolism (type II and type I collagen degradation) is increased by endurance exercise with intense joint loading, such as cross county and boat racing.

Thus, to test this hypothesis, we evaluated the cartilage and bone metabolism in collegiate athletes belonging to various sports clubs (soccer, tennis, triathlon, squash, swimming, volleyball, kendo, judo, gymnastics, basketball, handball, baseball, longdistance, throwing-event, jumping, sprint and futsal) by analyzing the urine levels of type II collagen degradation maker CTX-II and synthesis marker CPII, and type I collagen degradation marker NTx, and compared with those of non-athlete controls ${ }^{10)}$. Urine NTx and creatinine $(\mathrm{Cr})$ were measured by LSI Medience Corporation (Tokyo, Japan), based on an ELISA and an enzymatic assay, respectively. Urine CTX-II and

Table-1 Background of enrolled non-athletes and various sports athletes

\begin{tabular}{|c|c|c|c|c|c|}
\hline & & Ages (years) & Height $(\mathrm{cm})$ & Weight $(\mathrm{kg})$ & BMI $\left(\mathrm{kg} / \mathrm{m}^{2}\right)$ \\
\hline Non-athletes & $\mathrm{n}=10$ & $21.1 \pm 2.7$ & $171.0 \pm 6.0$ & $62.9 \pm 3.0$ & $21.6 \pm 1.3$ \\
\hline Soccer & $\mathrm{n}=10$ & $19.3 \pm 0.7$ & $176.2 \pm 5.2$ & $68.3 \pm 5.8$ & $22.0 \pm 1.4$ \\
\hline Tennis & $\mathrm{n}=9$ & $19.8 \pm 1.1$ & $173.5 \pm 5.2$ & $67.9 \pm 5.3$ & $22.5 \pm 1.4$ \\
\hline Triathlon & $\mathrm{n}=10$ & $20.3 \pm 0.9$ & $168.4 \pm 2.5$ & $62.0 \pm 4.9$ & $21.8 \pm 1.3$ \\
\hline Squash & $\mathrm{n}=11$ & $20.8 \pm 1.4$ & $168.2 \pm 5.8$ & $63.2 \pm 6.5$ & $22.3 \pm 1.9$ \\
\hline Swimming & $\mathrm{n}=5$ & $20.4 \pm 0.9$ & $179.8 \pm 2.2$ & $71.6 \pm 4.2$ & $22.1 \pm 0.8$ \\
\hline Volleyball & $\mathrm{n}=10$ & $19.0 \pm 0.7$ & $183.4 \pm 5.7$ & $76.9 \pm 6.5$ & $22.8 \pm 1.1$ \\
\hline Kendo & $\mathrm{n}=9$ & $20.4 \pm 0.5$ & $169.6 \pm 2.3$ & $66.0 \pm 3.6$ & $22.9 \pm 1.2$ \\
\hline Handball & $\mathrm{n}=10$ & $18.8 \pm 0.6$ & $172.7 \pm 5.5$ & $66.5 \pm 6.0$ & $22.3 \pm 1.9$ \\
\hline Baseball & $\mathrm{n}=10$ & $19.5 \pm 1.1$ & $172.6 \pm 6.1$ & $69.8 \pm 4.8$ & $23.4 \pm 1.0$ \\
\hline Gymnastics & $\mathrm{n}=10$ & $20.5 \pm 1.4$ & $166.2 \pm 4.7$ & $60.7 \pm 5.0$ & $22.0 \pm 1.1$ \\
\hline Judo & $\mathrm{n}=10$ & $18.7 \pm 0.7$ & $171.4 \pm 5.6$ & $79.7 \pm 13.8$ & $27.0 \pm 3.5$ \\
\hline Basketball & $\mathrm{n}=10$ & $20.3 \pm 1.3$ & $181.9 \pm 8.0$ & $76.1 \pm 7.9$ & $23.0 \pm 1.3$ \\
\hline Long-distance & $\mathrm{n}=10$ & $20.5 \pm 1.2$ & $167.3 \pm 6.3$ & $53.1 \pm 4.3$ & $19.0 \pm 0.6$ \\
\hline Sprint & $\mathrm{n}=7$ & $18.6 \pm 0.5$ & $173.3 \pm 4.9$ & $64.6 \pm 5.0$ & $21.5 \pm 1.0$ \\
\hline Jumping & $\mathrm{n}=10$ & $19.2 \pm 0.8$ & $175.6 \pm 4.3$ & $65.1 \pm 6.0$ & $21.1 \pm 1.4$ \\
\hline Throwing-event & $\mathrm{n}=9$ & $18.8 \pm 0.8$ & $178.0 \pm 4.6$ & $94.7 \pm 10.4$ & $29.9 \pm 3.0$ \\
\hline Futsal & $\mathrm{n}=10$ & $19.0 \pm 0.9$ & $171.1 \pm 4.5$ & $64.8 \pm 4.5$ & $22.2 \pm 1.5$ \\
\hline
\end{tabular}

Data represent the mean $\pm \mathrm{SD}$. 
CPII were measured using a Urine CartiLaps (CTX-II) EIA kit (Immunodiagnostic Systems Ltd., Boldon, UK) and a Procollagen II C-propeptide (CPII) ELISA kit (IBEX Pharmaceuticals Inc., Montreal, Canada), respectively.

Table-1 shows the background of enrolled nonathlete controls and athletes (all males). The ages of enrolled subjects were round 20 years. However, in their physical findings, volleyball and basketball players were taller than other athletes and nonathlete controls; the body weight of volleyball, judo, basketball and throwing-event players were heavier, whereas the body weight of long-distance runner were lighter than other athletes and non-athletes; thus, BMI of judo and throwingevent players was higher, whereas BMI of long-distance runner was lower than other athletes and non-athletes. Since physical findings were different among various sports players, the correlations of BMI with bone and cartilage metabolism markers were analyzed among enrolled subjects. Interestingly, there were negative correlations between BMI and the urine level of NTx, CTX-II or CPII (Figure-1), suggesting that bone and cartilage metabolism (such as type I collagen degradation, and type II collagen degradation and synthesis) is enhanced among athletes with lower BMI.

Next, NTx levels were compared among athletes of various sporting events. The results indicated that NTx levels were significantly higher in soccer, volleyball, basketball and handball players than non-athlete controls (Figure-2).

Furthermore, the levels of CTX-II and CPII, and CTX-II/CPII ratio were compared between non-athlete controls and athletes of various sporting events. Similar to the levels of NTx, CTX-II levels were significantly higher in soccer, volleyball and handball players than non-athlete controls (Figure-3A). In contrast, CPII levels were significantly higher in squash players and long-distance runners than non-athlete controls (Figure-3B). Base on the levels of CTX-II and CPII, the CTX-II/CPII ratios were calculated and compared between non-athlete controls and athletes of various sporting events. The results indicated that the CTX-II/CPII ratios were higher in soccer, volleyball, basketball and handball players than non-athlete controls (Figure-3C), suggesting that type II collagen degradation is relatively enhanced
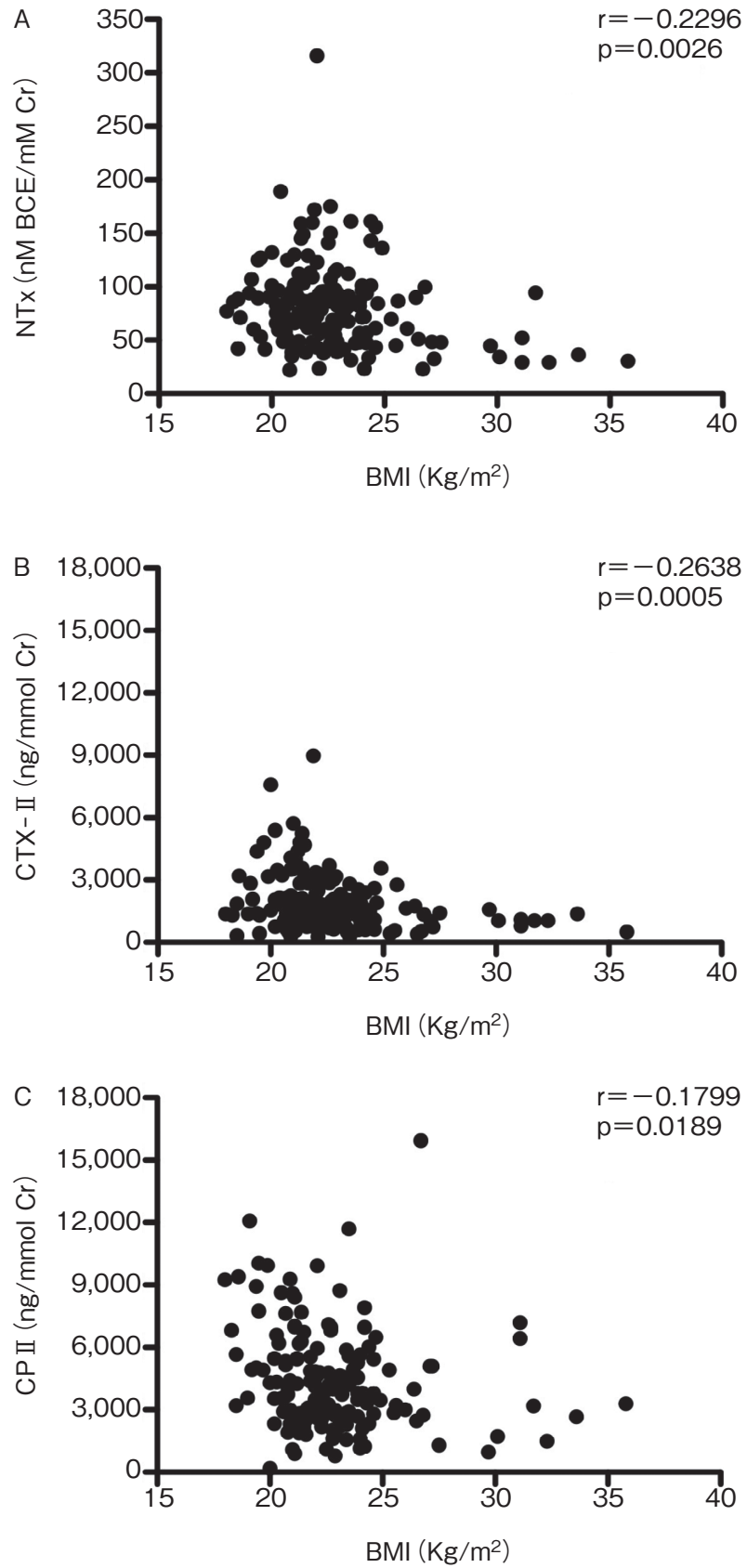

Figure-1 Correlation analysis of BMI and bone and cartilage metabolism markers

Urine levels of NTx, CTX-II and CPII in non-athlete controls and various sports athletes (shown in Table-1) were measured by ELISA and corrected by urinary creatinine $(\mathrm{Cr})$. Correlations between BMI and urine NTx (A), BMI and urine CTX-II (B), and BMI and CPII (C) were analyzed among enrolled subjects $(\mathrm{n}=170)$.

(Nagaoka I, et al: Functional Food Res, 2017; 13: 7-16 ${ }^{10)}$ )

compared with type II collagen synthesis in these athletes.

The similar changes of NTx (Figure-2) and CTX-II (Figure-3A) among various sports athletes indicate that NTx and CTX-II are likely to be 


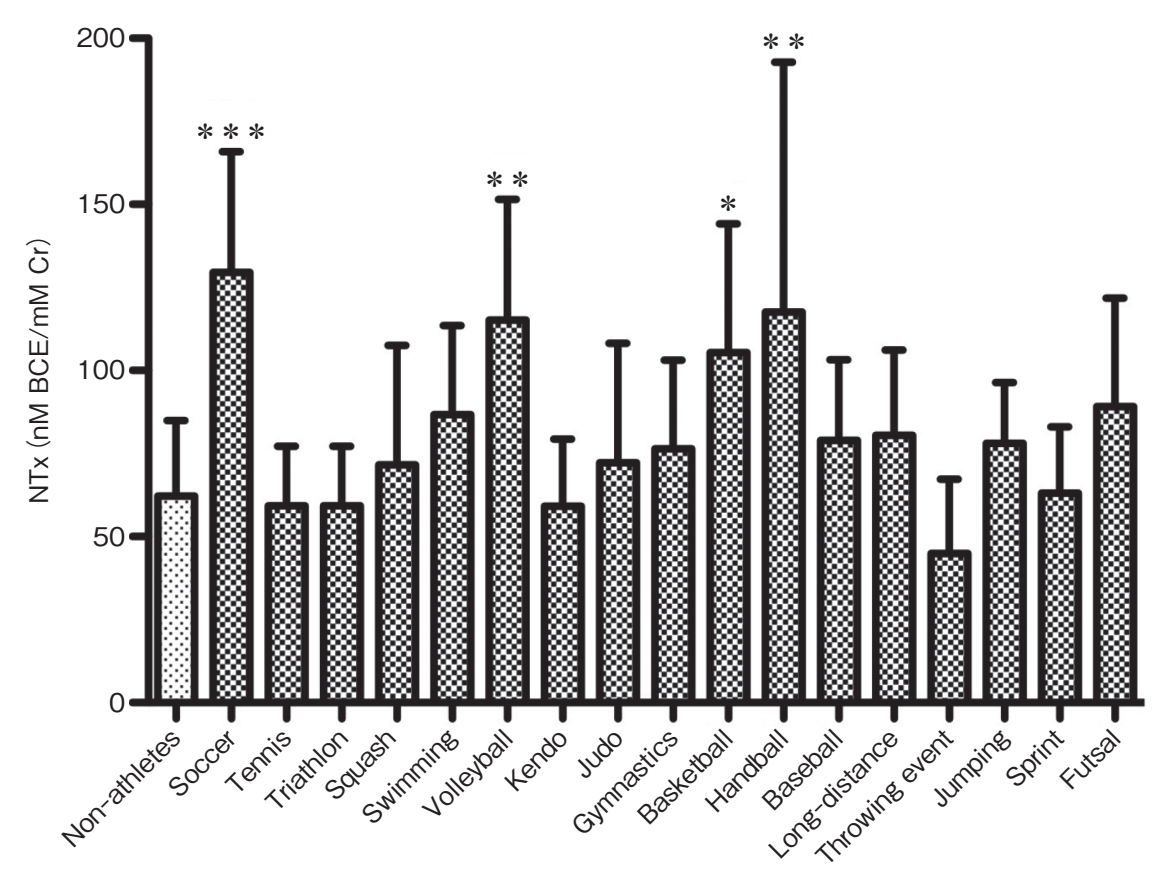

Figure-2 Comparison of the urine levels of NTx between non-athlete controls and various sports athletes Urine levels of NTx in non-athlete controls and various sports athletes (shown in Table-1) were measured by ELISA and corrected by urinary creatinine $(\mathrm{Cr})$. Data represent the mean $\pm \mathrm{SD}$. Values are compared between non-athletes $(10$ subjects) and various sports athletes $\left(5 \sim 11\right.$ subjects). ${ }^{*} \mathrm{p}<0.05,{ }^{* *} \mathrm{p}<0.01,{ }^{* * *} \mathrm{p}<0.001$

(Nagaoka I, et al: Functional Food Res, 2017; 13: 7-16 ${ }^{10)}$ )

changed in parallel within the body, although the two markers represent different bone and cartilage metabolism (type I collagen degradation and type II collagen degradation, respectively) ${ }^{5}$. To confirm this, we analyzed the correlation between NTx and CTX-II. As expected, the levels of NTx and CTX-II were significantly correlated (Figure-4A). In contrast, there was no significant correlation between NTx and CPII (Figure-4B), and CTX-II and CPII (Figure-4C). These observations indicate that type II collagen degradation (as assessed by CTX-II) is correlated with type I collagen degradation (as assessed by NTx) in sports athletes, and support our hypothesis that although both NTx and CTX-II are regarded as different bone and cartilage markers ${ }^{5}$, their levels are changed in parallel within the body.

\section{Conclusions}

In this study, we evaluated the cartilage and bone metabolism in college athletes belonging to various sports clubs by analyzing the levels of type II collagen degradation maker CTX-II and synthesis marker CPII, and type I collagen degradation marker NTx.

The results indicated that cartilage metabolism (type II collagen degradation) as well as bone metabolism (type I collagen degradation) is enhanced in athletes of ball games associated with jumping action, such as soccer, volleyball, basketball and handball.

We previously revealed that type II collagen degradation is enhanced in endurance athletes, such as soccer and rugby players (as evidenced by CTX-II) ${ }^{111}{ }^{12}$, and that glucosamine, a nutritional supplement, exhibits a chondroprotective action in these athletes by inhibiting type II collagen degradation, possibly via the suppression of matrix metalloproteinase (MMP) production ${ }^{13)-15)}$. Thus, it is interesting to speculate that glucosamine also exhibits a chondroprotective action in the athletes with jumping action and increased CTX-II level (found in this study, such as volleyball, basketball and handball players) by suppressing MMP production. However, the detailed action of glucosamine on the cartilage metabolism in these athletes remains to be elucidated in the future. 

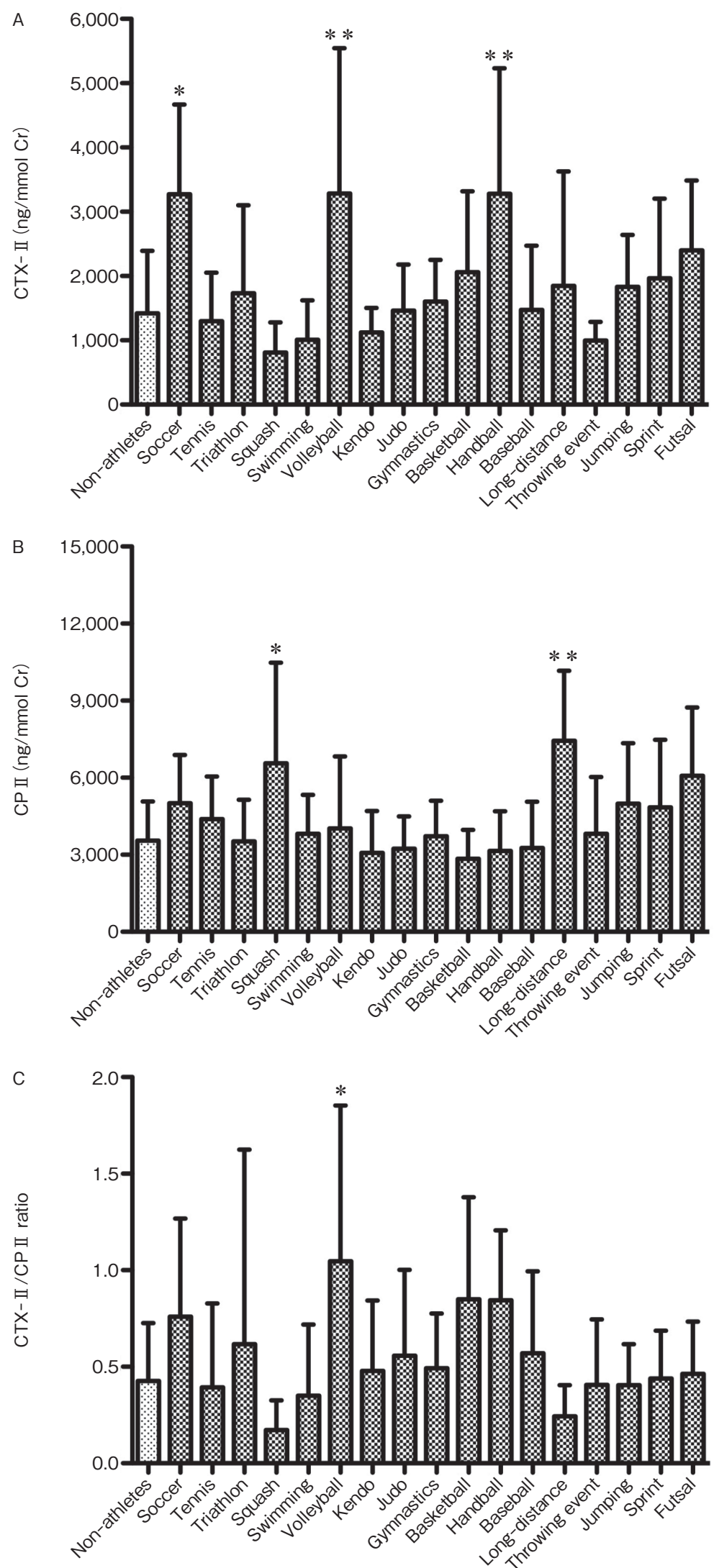

Figure-3 Comparison of the urine levels of CTX-II and CPII, and CTX-II/CPII ratio between non-athlete controls and various sports athletes

Urine levels of CTX-II (A) and CPII (B) in non-athlete controls and various sports athletes (shown in Table-1) were measured by ELISA and corrected by urinary creatinine (Cr). Moreover, the ratios of type II collagen degradation to synthesis (CTX-II/CPII) in non-athlete controls and various sports athletes were calculated (C), using the levels of CTX-II and CPII shown in Figures-3A and B. Data represent the mean \pm SD. Values are compared between non-athletes $\left(10\right.$ subjects) and various sports athletes $\left(5 \sim 11\right.$ subjects). ${ }^{*} \mathrm{p}<0.05,{ }^{* *} \mathrm{p}<0.01$ (Nagaoka I, et al: Functional Food Res, 2017; 13: 7-16 ${ }^{10)}$ ) 

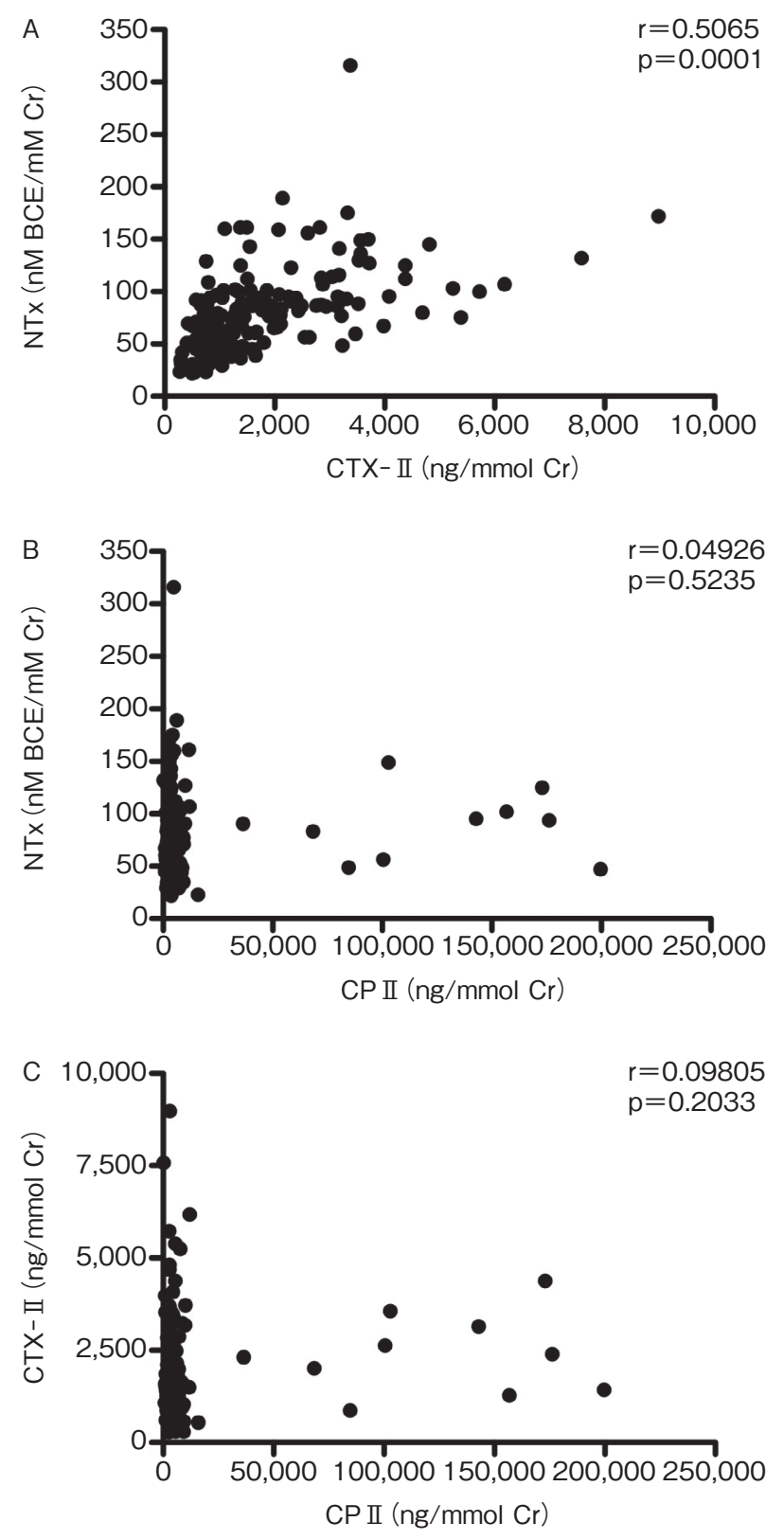

Figure-4 Correlation analysis of bone and cartilage metabolism markers

Correlations between CTX-II and NTx (A), CPII and NTx (B), and CPII and CTX-II (C) were analyzed among enrolled subjects $(\mathrm{n}=170$, non-athletes controls and various sports athletes, shown in Table-1).

(Nagaoka I, et al: Functional Food Res, 2017; 13: 7-16 ${ }^{10)}$ )

\section{References}

1) Roos H, Dahlberg L, Hoerrner LA, et al: Markers of cartilage matrix metabolism in human joint fluid and serum: the effect of exercise. Osteoarthritis Cartilage, 1995; 3: 7-14.

2) Qi C, Changlin H: Effects of moving training on histology and biomarkers levels of articular cartilage. J Surg Res, 2006; 135: 352-363.

3) O'Kane JW, Hutchinson E, Atley LM, Eyre DR: Sport-related differences in biomarkers of bone resorption and cartilage degradation in endurance athletes. Osteoarthritis Cartilage, 2006; 14: 71-76.

4) Buckwalter JA, Lane NE: Athletics and osteoarthritis. Am J Sports Med, 1997; 25: 873-881.

5) Rousseau JC, Delmas PD: Biological markers in osteoarthritis. Nat Clin Pract Rheumatol, 2007; 3: 346-356.

6) Poole AR: Biochemical/immunochemical biomarkers of osteoarthritis: utility for prediction of incident or progressive osteoarthritis. Rheum Dis Clin North Am, 2003; 29: 803-818.

7) Christgau S, Garnero P, Fledelius C, et al: Collagen type II C-telopeptide fragments as an index of cartilage degradation. Bone, 2001; 29: 209-215.

8) Poole AR, Ionescu M, Fitzcharles MA, Billinghurst RC: The assessment of cartilage degradation in vivo: development of an immunoassay for the measurement in body fluids of type II collagen cleaved by collagenases. J Immunol Methods, 2004; 294: 145-153.

9) Shinmei M, Ito K, Matsuyama S, Yoshihara Y, Matsuzawa K: Joint fluid carboxy-terminal type II procollagen peptide as a marker of cartilage collagen biosynthesis. Osteoarthritis Cartilage, 1993; 1: 121-128.

10) Nagaoka I, Yoshimura M, Sakamoto K, Tsuruta A: Evaluation of cartilage and bone metabolism in osteoarthritic patients and collegiate athletes belonging to various sports clubs by using type II collagen degradation and synthesis markers, and type I collagen degradation marker. Functional Food Res, 2017; 13: 7-16. (in Japanese)

11) Yoshimura M, Sakamoto K, Tsuruta A, et al: Evaluation of the effect of glucosamine administration on biomarkers for cartilage and bone metabolism in soccer players. Int J Mol Med, 2009; 24: 487-494.

12) Tsuruta A, Nagaoka I: Evaluation of a jelly-type functional food on the cartilage metabolism in male professional rugby players. Functional Food Res, 2016; 12: $39-41$.

13) Nakamura H, Shibakawa A, Tanaka M, Kato T, Nishioka $\mathrm{K}$ : Effects of glucosamine hydrochloride on the production of prostaglandin $\mathrm{E}_{2}$, nitric oxide and metalloproteases by chondrocytes and synoviocytes in osteoarthritis. Clin Exp Rheumatol, 2004; 22: 293-299.

14) Derfoul A, Miyoshi AD, Freeman DE, Tuan RS: Glucosamine promotes chondrogenic phenotype in both chondrocytes and mesenchymal stem cells and inhibits MMP-13 expression and matrix degradation. Osteoarthritis Cartilage, 2007; 15: 646-655.

15) Nakamura H, Masuko K, Yudoh K, Kato T, Kamada T, Kawahara T: Effects of glucosamine administration on patients with rheumatoid arthritis. Rheumatol Int, 2007; 27: 213-218. 